\title{
Cardiovascular Pharmacological Support Among Preterm Infants in Chinese Referral Center Neonatal Intensive Care Units
}

OPEN ACCESS

Edited by:

Christoph Bührer,

Charité - Universitätsmedizin

Berlin, Germany

Reviewed by:

Lai Wen Yu,

University of Hong Kong, China

Kurt Schibler,

Cincinnati Children's Hospital Medical

Center, United States

*Correspondence:

Yun Cao

yuncao@fudan.edu.cn

†These authors have contributed equally to this work

¥Present address:

Ningxin Luo,

Shanghai Children's Medical Center,

Shanghai JiaoTong University School

of Medicine, Shanghai, China

$\S_{A}$ full list of REIN-EPIQ Writing Study

Group can be found in the

Supplementary Materia

Specialty section:

This article was submitted to

Neonatology,

a section of the journal

Frontiers in Pediatrics

Received: 07 December 2020 Accepted: 29 March 2021

Published: 22 April 2021

Citation:

Luo N, Jiang S, McNamara PJ, Li X,

Guo $Y$, Wang $Y$, Han J, Deng $Y$,

Yang Y, Lee SK and Cao Y (2021)

Cardiovascular Pharmacological

Support Among Preterm Infants in

Chinese Referral Center Neonatal Intensive Care Units.

Front. Pediatr. 9:638540.

doi: 10.3389/fped.2021.638540

\begin{abstract}
Ningxin Luo ${ }^{1 \neq}$, Siyuan Jiang ${ }^{1 \dagger}$, Patrick J. McNamara ${ }^{2}$, Xiaoying $\mathrm{Li}^{3}$, Yan Guo ${ }^{4}$, Yang Wang ${ }^{5}$, Junyan Han ${ }^{1}$, Yingping Deng ${ }^{1}$, Yi Yang ${ }^{6}$, Shoo K. Lee ${ }^{7,8,9}$ and Yun Cao ${ }^{*}$ on behalf of the REIN-EPIQ Study Group ${ }^{\S}$

${ }^{1}$ Children's Hospital of Fudan University, Shanghai, China, ${ }^{2}$ Department of Pediatrics and Internal Medicine, University of lowa, lowa City, IA, United States, ${ }^{3}$ Qilu Children's Hospital of Shandong University, Jinan, China, ${ }^{4}$ Children's Hospital of Nanjing Medical University, Nanjing, China, ${ }^{5}$ The First Affiliated Hospital of Anhui Medical University, Hefei, China, ${ }^{6}$ National Health Commision (NHC) Key Laboratory of Neonatal Diseases (Fudan University), Children's Hospital of Fudan University, Shanghai, China, ${ }^{7}$ Maternal-Infant Care Research Centre and Department of Pediatrics, Mount Sinai Hospital, Toronto, ON, Canada, ${ }^{8}$ Department of Pediatrics, University of Toronto, Toronto, ON, Canada, ${ }^{9}$ Department of Obstetrics and Gynecology, Dalla Lana School of Public Health, University of Toronto, Toronto, ON, Canada
\end{abstract}

Objective: To describe cardiovascular pharmacological support in infants born at $<34$ weeks' gestation within the first postnatal week in Chinese neonatal intensive care units (NICUs).

Design: A secondary analysis of data from a multicenter randomized controlled study (REIN-EPIQ). A questionnaire regarding cardiovascular support practices was also completed by all participating NICUs.

Setting: Twenty-five tertiary hospitals from 19 provinces in China.

Patients: All infants born at $<34$ weeks' gestation and admitted to participating NICUs within the first postnatal week from May 2015 to April 2018 were included. Infants who were discharged against medical advice were excluded.

Measures and Main Results: Among the 26,212 preterm infants $<34$ weeks, $16.1 \%$ received cardiovascular pharmacological support. The use rates increased with decreasing gestational age and birth weight, with $32.5 \%$ among infants $<28$ weeks and $35.9 \%$ among infants $<1,000 \mathrm{~g}$. Cardiovascular pharmacological support was independently associated with higher risks of death (aOR 2.8; 95\% Cl 2.4-3.3), severe intraventricular hemorrhage $(\mathrm{IVH})(\mathrm{aOR} 2.1 ; 95 \% \mathrm{Cl} 1.8-2.5)$ and bronchopulmonary dysplasia (BPD) (aOR 2.2; 95\% Cl 2.0-2.5). Overall 63.1\% courses of cardiovascular pharmacological support were $>3$ days. Prolonged cardiovascular pharmacological support (>3 days) was independently associated with lower rates of survival without morbidity in very-low-birth-weight infants, compared with infants with shorter durations. Dopamine was the most commonly used cardiovascular agent. The cardiovascular pharmacological support rates varied from 1.9 to $65.8 \%$ among the participating NICUs.

Conclusions: The rate of cardiovascular pharmacological support within the first postnatal week was high with prolonged durations in Chinese NICUs. Marked 
variation in cardiovascular support existed among participating NICUs. Cardiovascular pharmacological support during the early postnatal period, especially prolonged, may be associated with adverse neonatal outcomes.

Clinical Trial Registration: The original trial was registered as "Reduction of Infection in Neonatal Intensive Care Units using the Evidence-based Practice for Improving Quality" (ID: NCT02600195) on clinicaltrials.gov. https://clinicaltrials.gov/ct2/ show/NCT02600195?term=NCT02600195\&draw=2\&rank=1.

Keywords: preterm infants, neonate, cardiovascular pharmacological support, neonatal intensive care units, China, outcome assessment

\section{BACKGROUND}

Cardiovascular pharmacological support is among the most commonly used therapies in NICUs, especially in preterm infants (1). The first few days after birth represent a unique period of life as the dynamics of the circulatory system change for adaptation to an extra-uterine environment (2). During this vulnerable period, preterm infants are at high risk of systemic hypotension. The sudden increase in systemic vascular resistance leading to transient myocardial dysfunction, which prompts the need for cardiovascular pharmacological support, is thought to be a major contributor to low cardiac output and suboptimal oxygen delivery to the brain (3). There is no consensus, however, on the definition of an acceptable blood pressure; therefore, wide variations in intervention thresholds, medication selection, duration, and dosage of cardiovascular pharmacological support exist $(4,5)$. In addition, it is unclear whether cardiovascular pharmacological support during the early stage of life could improve the outcomes of preterm infants (6-9). Data on cardiovascular pharmacological support in Chinese NICUs and outcomes among infants who received drugs have not been reported. The primary aim of this study was to describe the use of cardiovascular pharmacological support during the first postnatal week among the largest contemporary cohort of infants born at $<34$ weeks gestation in Chinese NICUs.

\section{METHODS}

\section{Study Design}

The study was a secondary analysis of data from a multicenter cluster randomized controlled study named "Reduction of Infection in Neonatal Intensive Care Units using the Evidencebased Practice for Improving Quality (REIN-EPIQ)," which was registered at clinicaltrials.gov (NCT0260015) $(10,11)$. Twentyfive tertiary hospitals from 19 provinces in China participated in this study. These tertiary NICUs represent the highest level of

\footnotetext{
Abbreviations: NICUs, neonatal intensive care units; IVH, intraventricular hemorrhage; BPD, bronchopulmonary dysplasia; PDA, patent ductus arteriosus; TRIPS, transport risk index of physiologic stability score; PVL, periventricular leukomalacia; ROP, retinopathy of pre-maturity; EOS, early onset sepsis; LOS, late onset sepsis; NEC, necrotizing enterocolitis; EPI, extremely pre-mature infants; VLBW, very low birth weight infants; ELBW, extremely low birth weight infants; TnECHO, targeted neonatal echocardiography; NNN, Norwegian Neonatal Network; CNN, Canadian Neonatal Network.
}

neonatal care in their regions. The study was approved by the Ethics Committee of the Children's Hospital of Fudan University [approval number (2015) 28] for all sites.

\section{Study Population}

All infants born at $<34$ weeks gestation between May 1st, 2015, and April 30th, 2018, and admitted to 25 participating NICUs within the first postnatal week were enrolled. Infants who were discharged against medical advice and did not receive complete care within the first postnatal week were excluded.

\section{Data Collection}

Detailed clinical data, including data regarding duration of cardiovascular pharmacological support, were collected prospectively by trained data abstractors using a standardized database. All data collection followed standard operations and definitions.

\section{Variable Definitions}

Cardiovascular pharmacological support was defined as continuous intravenous infusion of at least one of the following medications: dopamine, dobutamine, epinephrine, norepinephrine or milrinone. Detailed information, such as the dose of the medication, was not collected. The transport risk index of physiologic stability (TRIPS) score (12), a critical illness score developed by the Canadian Neonatal Network, was used to represent the illness severity on admission. Early onset sepsis (EOS) was defined as sepsis occurring in 3 days of age. Extremely pre-mature infants (EPI) and very pre-mature infants were defined as infants born with gestational age $<28^{+0}$ weeks and $28^{+0}-31^{+6}$ weeks, respectively. Very-low-birth-weight (VLBW) and Extremely-low-birth-weight (ELBW) infants were defined as infants born with a birth weight $<1,500$ and $<1,000$ g, respectively.

\section{Outcomes}

The primary outcome was a composite of one of six major morbidities including periventricular leukomalacia (PVL), intraventricular hemorrhage (IVH), retinopathy of pre-maturity (ROP), bronchopulmonary dysplasia (BPD), late onset sepsis (LOS) or necrotizing enterocolitis (NEC). "Survival without morbidity" was defined as surviving infants who were not diagnosed with any of these major morbidities at discharge. PVL was defined as the presence of periventricular cysts on 
cranial ultrasound or magnetic resonance imaging scans before discharge. IVH was detected by ultrasound findings and was defined according to the criteria of Papile et al. (13). Severe IVH was defined as grade III or IV IVH. ROP was defined according to the International Classification of ROP (14). Severe ROP was defined as ROP $\geq$ stage 3 . BPD was defined as mechanical ventilation or oxygen dependency at 36 weeks post-menstrual age or discharge (15). LOS was defined as blood culture-proven sepsis occurring after 3 days of life $(16,17)$. NEC was defined as stage II or above NEC according to Bell's criteria (18). Survival without morbidity was defined as infants who survived at discharge without being diagnosed with any of the six major morbidities.

\section{Survey of Intervention Thresholds and Cardiovascular Pharmacological Support}

To evaluate clinical practices of cardiovascular pharmacological support for preterm infants in different NICUs, a questionnaire was developed and filled out as a specific addition by the NICU director or a senior neonatologist according to the practice guidelines (if existed) or general practices in the individual NICU. The contents of the questionnaire included (i) the diagnostic criteria of hypotension; (ii) the most commonly used cardiovascular pharmacological support; (iii) indications for cardiovascular pharmacological support; (iv) indications for discontinuing cardiovascular pharmacological support; (v) methods of blood pressure measurement and circulation status evaluation. The questionnaire can be found as Supplementary Material.

\section{Statistical Analysis}

Infant characteristics and outcomes were compared between the cardiovascular pharmacological support and control groups by Student's $t$-tests for continuous variables and Chi-square-tests for categorical variables. A multivariate logistic regression model was used to compare outcomes between the two groups adjusting for relevant confounders, including gestational age, male sex, being small for gestational age, 1-min Apgar score $\leq 3 \mathrm{~min}$, cesarean section, antenatal steroids, prenatal care, maternal hypertension, maternal diabetes, primigravida, inborn status and TRIPS score. Variations in cardiovascular pharmacological support among the participating NICUs were also examined by multivariate logistic regression adjusting for the same relevant confounders. An important consideration was the fact that the duration of cardiovascular pharmacological support could be abbreviated by death. Therefore, we performed additional subgroup analyses to compare morbidity rates in survivors who received cardiovascular pharmacological support for $\leq 3$ days and those who received therapy for $>3$ days. Separate analyses were performed for extremely-low-birth-weight (ELBW) infants (birth weight $<1,000 \mathrm{~g}$ ), very-low-birth-weight (VLBW) infants (birthweight 1,000-1,500 g) and infants with birth weights $\geq 1,500$ g. Stata version 15.0 (StataCorp LP, USA) was used for statistical analysis. Statistical significance was set as a $p$-value of $<0.05$.

\section{RESULTS}

\section{Infant Characteristics}

A total of 27,532 preterm infants born at a gestational age $<34$ weeks were admitted to participating NICUs during the study period. After excluding 1,320 infants who were discharged against medical advice within the first week of life, the remaining 26,212 infants constituted the study population. The median gestational age and weight at birth were 31.3 (IQR: 30.133.0) weeks and 1,629 (IQR: 1,340-1,900) grams, respectively. Extremely pre-mature infants (EPI, $<28$ weeks) and ELBW infants accounted for $6.2 \%(1,622)$ and $5.4 \%(1,432)$ of the sample, respectively.

\section{Cardiovascular Pharmacological Support Rate}

Overall, $16.1 \%(4,226 / 26,212)$ of infants received cardiovascular pharmacological support during the first week. The rates of cardiovascular pharmacological support in EPI and ELBW

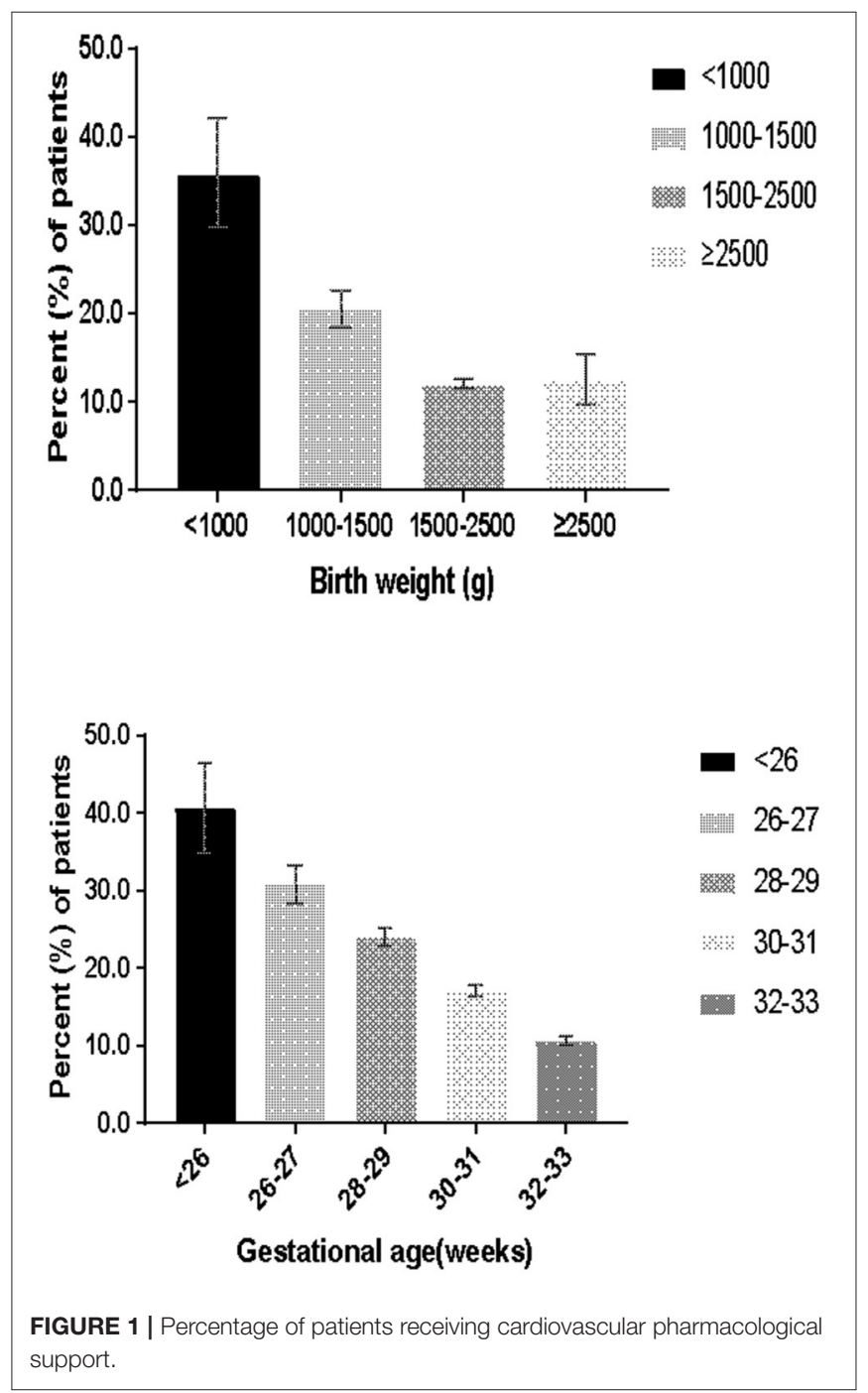


infants were $32.5 \%(527 / 1,622)$ and $35.9 \% \quad(514 / 1,432)$, respectively. The proportion of preterm infants who received cardiovascular pharmacological support decreased with increasing gestational age and birth weight (Figure 1). Overall, $89.6 \%(3,788 / 4,226)$ of cardiovascular pharmacological support were initiated within 3 days, and $58.4 \%(2,470 / 4,226)$ were initiated on the first day.

Infants who received cardiovascular pharmacological support had lower gestational ages, lower birth weights, lower 1 and 5min Apgar scores and higher TRIPS scores than infants who were not treated with these drugs. Infants receiving cardiovascular pharmacological support were also more likely to be male and to be small for their gestational age (Table 1).

\section{Trends in Cardiovascular Pharmacological Support Rate}

Over the study period, there was a decreasing trend of cardiovascular pharmacological support each year. The overall cardiovascular pharmacological support rate was $18.1 \%$ $(1,359 / 7,500), 15.9 \%(1,531 / 9,642)$, and $14.7 \%(1,336 / 9,070)$ in the first, second and third study years $\left(\chi^{2}=35.5612, p<\right.$ $0.001)$, respectively. The specific annual rate of cardiovascular pharmacological support decreased among EPI (36.3 vs. $32.4 \%$ vs. $30.5 \%, p=0.15)$.

\section{Outcomes of Infants}

The univariate analysis revealed that infants who received cardiovascular pharmacological support had higher rates of death, NEC, severe IVH, PVL, ROP, BPD, and LOS. After adjusting for relevant confounders, cardiovascular pharmacological support within the first postnatal week was independently associated with higher risks of death (aOR 3.0; 95\% CI: 2.6-3.5), severe IVH (aOR 2.1; 95\% CI: 1.8-2.4), and BPD (aOR 2.2; 95\% CI: 2.0-2.5) (Table 2).

\section{Treatment Duration and Outcomes}

Among the 2,470 infants in whom cardiovascular pharmacological support was initiated on the first day of life, 2,450 (99.2\%) had a known duration. In these patients, $5.8 \%$ $(143 / 2,450), 16.4 \%(401 / 2,450), 14.7 \%(359 / 2,450)$, and $63.1 \%$ $(1,547 / 2,450)$ received cardiovascular pharmacological support for 1, 2, 3 days, or more than 3 days, respectively. In total, 295 infants died during hospitalization, of whom 227 (76.9\%) died while cardiovascular pharmacological treatment, leading to an abbreviated duration of therapy. Among survivors, infants who received cardiovascular pharmacological support for $>3$ days had lower rates of survival without morbidity (66.7 vs. $75.4 \%, p$ $<0.001)$ and higher rates of severe IVH (9.8 vs. $4.8 \%, p<0.001$ ) and BPD (18.7 vs. $14.1 \%, p=0.01)$ compared with infants who received cardiovascular pharmacological support for $\leq 3$ days (Table 3). After adjustment, a treatment duration $>3$ days was independently associated with lower rates of survival without morbidity among VLBW ( $p=0.04$; Table 4$)$.

\section{Discrepancy in Cardiovascular Pharmacological Support at Different NICUs}

The rate of cardiovascular pharmacological support varied from 1.9 to $65.8 \%$ among the participating NICUs $\left(\chi^{2}=5,100, p<\right.$ 0.001 ) (Figure 2A). After adjusting for relevant confounders, the magnitude of variation remained significant (Figure 2B).

All participating centers responded to the questionnaire. The criteria to initiate cardiovascular pharmacological support varied significantly among NICUs. The most common indicators for treatment included hypotension $(24 / 25,96 \%)$, prolonged capillary refill time $(22 / 25,88 \%)$, persistent metabolic acidosis $(18 / 25,72 \%)$, low urine output $(17 / 25,68 \%)$, elevated lactate value $(15 / 25,60 \%)$ and diminished color of peripheries $(14 / 25$, $56 \%)$. Twenty NICUs (80\%) defined hypotension as a mean blood pressure in $\mathrm{mmHg}$ less than the gestational age in weeks. The other definitions used were blood pressure $<30 \mathrm{mmHg}$ or blood pressure (in $\mathrm{mmHg}$ ) below the 10th percentile for age and gender. All NICUs had the ability to perform invasive blood pressure monitoring and bedside cardiac ultrasound; however, targeted neonatal echocardiography (TnECHO) was not used in Chinese NICUs to assist in clinical decision making in the study period. In 22 NICUs (88\%), dopamine alone was the first line drug, two hospitals used dopamine in combination with dobutamine, and one hospital used three agents together (dopamine, dobutamine, and milrinone) as the first line treatment.

\section{DISCUSSION}

To our knowledge, this is the largest contemporary cohort of preterm infants and the first study to evaluate cardiovascular pharmacological support in Chinese NICUs. Our results showed higher cardiovascular pharmacological support rates than those of the Norwegian Neonatal Network (NNN) and the Canadian Neonatal Network (CNN), suggesting that Chinese NICUs might overuse cardiovascular pharmacological support (19). Neonates who received cardiovascular pharmacological support, particularly those who received treatment for more than 3 days, were more likely to die or develop neonatal morbidity.

Exclusive reliance on blood pressure as the singular metric of hemodynamic stability, a lack of consensus on definition of hypotension and a lack of consensus on thresholds for therapeutic intervention led to marked variation in cardiovascular pharmacological support. Several studies have demonstrated variation in cardiovascular pharmacological support among different centers (19-21). A recent US study showed that cardiovascular pharmacological support rates ranged from 4 to $39 \%$ among six NICUs (22). Data from NNN (23) and CNN (19) also showed marked variations in cardiovascular pharmacological support rates ranging between $1-8 \%$ and $0-36 \%$ among different NICUs, respectively. Our results demonstrated the highest order of magnitude variation, specifically, a 35 -fold difference $(1.9-65.8 \%)$ in drug use among Chinese NICUs. A lack of reliable normative blood pressure data in preterm infants, non-judicious use 
TABLE 1 | Characteristics of the study population.

\begin{tabular}{|c|c|c|c|}
\hline Characteristics & $\begin{array}{l}\text { Cardiovascular pharmacological support } \\
\text { group, } \\
(N=4,226)\end{array}$ & $\begin{array}{l}\text { No cardiovascular pharmacological support } \\
\text { group, } \\
(N=21,986)\end{array}$ & $p$-value \\
\hline$<26^{+0}$ weeks', $n / N(\%)$ & $113 / 278(40.7)$ & $165 / 278(59.4)$ & \\
\hline $26^{+0}-27^{+6}$ weeks', $n / N(\%)$ & $414 / 1,344(30.8)$ & $930 / 1,344(69.2)$ & \\
\hline $28^{+0}-29^{+6}$ weeks', $n / N(\%)$ & $1,006 / 4,187(24.0)$ & $3,181 / 4,187(76.0)$ & \\
\hline $32^{+0}-33^{+6}$ weeks', $n / N(\%)$ & $1,346 / 12,513(10.8)$ & $11,167 / 12,513(89.2)$ & \\
\hline Birth weight (grams), median (IQR) & $1,481(1,170-1,760)$ & $1,657(1,380-1,940)$ & $<0.001$ \\
\hline$<1,000$ grams, $n / N(\%)$ & $514 / 1,432(35.9)$ & $918 / 1,432(64.1)$ & \\
\hline $1,000-1,499$ grams, $n / N(\%)$ & 1,759/8,573 (20.5) & $6,814 / 8,573(79.5)$ & \\
\hline $1,500-2,500$ grams, $n / N(\%)$ & $1,887 / 15,670(12.0)$ & 13,783/15,670 (88.0) & \\
\hline 5-min Apgar $\leq 3^{b}, n / N(\%)$ & $105 / 3,842(2.7)$ & $142 / 20,150(0.7)$ & $<0.001$ \\
\hline Cesarean section, $n / N(\%)$ & 2,266/4,222 (53.7) & $12,185 / 21,976(55.5)$ & 0.03 \\
\hline Antenatal steroids ${ }^{c}, n / N(\%)$ & 2,653/3,948 (67.2) & 13,929/20,932 (66.5) & 0.42 \\
\hline Prenatal care $^{\mathrm{d}}, n / N(\%)$ & $4,124 / 4,182(98.6)$ & $21,504 / 21,789$ (98.7) & 0.68 \\
\hline Maternal hypertension ${ }^{\mathrm{e}}, n / N(\%)$ & $693 / 4,162(16.7)$ & $3,691 / 21,790(16.9)$ & 0.65 \\
\hline Maternal diabetes ${ }^{f}, n / N(\%)$ & $574 / 4,164(13.8)$ & $2,577 / 21,769(11.8)$ & $<0.001$ \\
\hline Primigravida $9, n / N(\%)$ & $1,356 / 4,223(32.1)$ & 8,029/21,966 (36.6) & $<0.001$ \\
\hline Inborn status, $n / N(\%)$ & 2,691/4,226 (63.7) & $15,610 / 21,986(71.0)$ & $<0.001$ \\
\hline TRIPS score ${ }^{\text {h }}$, median (IQR) & $19(13-28)$ & $12(6-19)$ & $<0.001$ \\
\hline Invasive mechanical ventilation, $n / N(\%)$ & $2,571 / 4,226(60.8)$ & $4,757 / 21,986(21.6)$ & $<0.001$ \\
\hline
\end{tabular}

IQR, interquartile range; TRIPS, Transport Risk Index of Physiologic Stability; EOS, early-onset sepsis.

${ }^{a}$ Data on 1-min Apgar score were missing in 1,004 infants.

${ }^{b}$ Data on 5-min Apgar score were missing in 2,220 infants.

${ }^{\circ}$ Data on antenatal steroids were missing in 1,332 infants.

${ }^{d}$ Data on prenatal care were missing in 241 infants.

${ }^{e}$ Data on maternal hypertension were missing in 260 infants.

${ }^{f}$ Data on maternal diabetes were missing in 279 infants.

${ }^{9}$ Data on primigravida were missing in 23 infants.

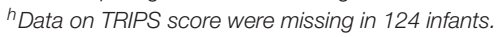

of cardiovascular pharmacological support, and diagnostic imprecision related to poor access to TnECHO services may contribute to variations in practice (19-21, 24-26). Institutional habits and culture regarding cardiovascular pharmacological support may also play a role in the variation of use rate.

In recent decades, with the development of perinatal care, the number of extremely preterm infants and very preterm infants cared in Chinese NICUs has increased significantly and the experience in treatment of preterm infants have been accumulated, which may contribute to the decreasing trend of cardiovascular pharmacological support reported in our study. Previous studies have shown that antenatal corticosteroids are crucial treatment in improving neonatal outcomes and decreasing mortality for those preterm infants (27), which may be helpful to prevent the need for cardiovascular support. Although still lower than developed countries, it is still inspiriting to see an increasing trend of antenatal steroid use during the past a few years $(11,28,29)$. More evidence-based practices regarding resuscitation and initial stabilization have been adopted in Chinese perinatal centers, such as delayed cord clamping and proper temperature control (30). On the other hand, from our daily clinical experience, we have observed that the concept of "treating the baby instead of treating the number (such as blood pressure)" has been increasing recognized by neonatologists in China, which may help to decrease the unnecessary use of cardiovascular pharmacological support. Further investigations are needed to interpret the reason for the decreasing trend and further optimize cardiovascular pharmacological support in Chinese NICUs. 
TABLE 2 | Outcomes of infants and cardiovascular pharmacological support.

\begin{tabular}{|c|c|c|c|c|}
\hline Outcomes & $\begin{array}{c}\text { Cardiovascular } \\
\text { pharmacological support } \\
\text { group, } N=4,226\end{array}$ & $\begin{array}{c}\text { No cardiovascular } \\
\text { pharmacological support } \\
\text { group, } N=21,986\end{array}$ & $\begin{array}{l}\text { Unadjusted odds ratio } \\
\qquad(95 \% \mathrm{Cl})\end{array}$ & Adjusted odds ratiob $(95 \% \mathrm{Cl})$ \\
\hline Death in first 7 days, $n / N(\%)$ & 424/4,226 (10.0) & $320 / 21,986(1.5)$ & $7.6(6.5-8.8)$ & $4.2(3.6-5.1)$ \\
\hline Death, $n / N(\%)$ & $565 / 4,226(13.4)$ & $621 / 21,986(2.8)$ & $5.3(4.7-6.0)$ & $3.0(2.6-3.5)$ \\
\hline NEC, $n / N(\%)$ & $181 / 4,226$ (4.3) & $779 / 21,986(3.5)$ & $1.2(1.0-1.4)$ & $0.9(0.8-1.1)$ \\
\hline Severe $\mathrm{IVH}^{\mathrm{C}}, n / N(\%)$ & $385 / 3,814(10.1)$ & $739 / 20,191(3.7)$ & $3.0(2.6-3.4)$ & $2.1(1.8-2.4)$ \\
\hline$P V L^{d}, n / N(\%)$ & $134 / 3,656(3.7)$ & $479 / 19,041(2.5)$ & $1.5(1.2-1.8)$ & $1.0(0.8-1.3)$ \\
\hline ROP, $n / N(\%)$ & 492/4,226 (11.6) & 1,897/21,986 (8.6) & $1.4(1.3-1.6)$ & $0.7(0.6-0.8)$ \\
\hline $\mathrm{ROP} \geq$ stage $3, n / N(\%)$ & $68 / 4,226(1.6)$ & $149 / 21,986(0.7)$ & $2.4(1.8-3.2)$ & $1.1(0.8-1.5)$ \\
\hline BPD, $n / N(\%)$ & $1,149 / 4,226(27.2)$ & 2,098/21,986 (9.5) & $3.5(3.3-3.8)$ & $2.2(2.0-2.5)$ \\
\hline $\operatorname{LOS}^{\mathrm{e}}, n / N(\%)$ & $244 / 4,224(5.8)$ & $883 / 21,982$ (4.0) & $1.5(1.3-1.7)$ & $1.1(0.9-1.3)$ \\
\hline
\end{tabular}

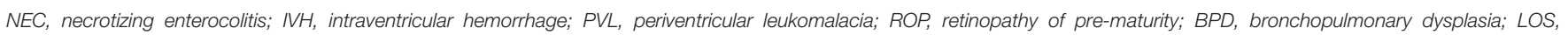
culture-proven late onset sepsis.

alnfants in no vasopressor group served as reference.

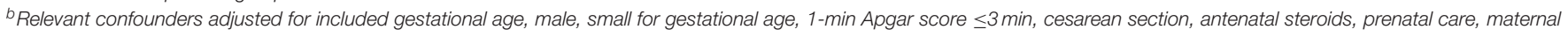
hypertension, maternal diabetes, primigravida, inborn status, and TRIPS score.

cIncidence of PVL or IVH was calculated among infants with neuroimaging results. Data on severe IVH were missing in 2,207 infants.

${ }^{d}$ Incidence of PVL or IVH was calculated among infants with neuroimaging results. Data on PVL were missing in 3,515 infants.

e Data on LOS were missing in 6 infants.

TABLE 3 | Outcomes and cardiovascular pharmacological support duration among survivors.

\begin{tabular}{|c|c|c|c|c|}
\hline Total & $\begin{array}{c}\text { Duration } \leq 3 \text { days } \\
\qquad N=722\end{array}$ & $\begin{array}{l}\text { Duration }>3 \text { days } \\
\qquad N=1,433\end{array}$ & $\begin{array}{l}\text { Odds ratio } \\
(95 \% \mathrm{Cl})\end{array}$ & $p$-value \\
\hline Survival without morbidity, $n / N(\%)$ & $544 / 722(75.4)$ & $956 / 1,433(66.7)$ & $1.52(1.24-1.88)$ & $<0.001$ \\
\hline NEC, $n / N(\%)$ & 24/722 (3.3) & $54 / 1,433(3.8)$ & $0.88(0.51-1.46)$ & 0.60 \\
\hline Severe IVH ${ }^{a}, n / N(\%)$ & 32/673 (4.8) & 136/1,394 (9.8) & $0.46(0.30-0.69)$ & $<0.001$ \\
\hline$P V L^{b}, n / N(\%)$ & $18 / 654(2.8)$ & $57 / 1,370(4.2)$ & $0.65(0.36-1.14)$ & 0.117 \\
\hline BPD, $n / N(\%)$ & 102/722 (14.1) & 268/1,433 (18.7) & $0.72(0.55-0.92)$ & 0.01 \\
\hline LOS, n/N (\%) & 30/722 (4.2) & 89/1,433 (6.2) & $0.65(0.41-1.01)$ & 0.049 \\
\hline
\end{tabular}

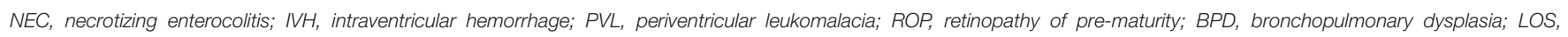
culture-proven late onset sepsis.

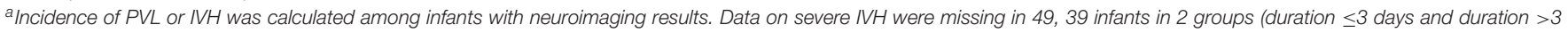
days), respectively.

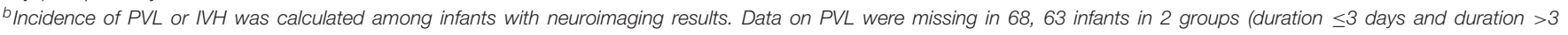
days), respectively.

We can also see a higher rate of cardiovascular pharmacological support in infants diagnosed with EOS which is reasonable by providing inotropic actions as well as decreasing systemic vascular resistance. Conventionally, dopamine is the first-line vasoactive drug in neonatal septic shock which is used empirical and extrapolated from adult and pediatric data mostly. It is unclear whether the treatment of low blood pressure results in any improvement of clinical outcomes or long-term neurological results. Previous studies have highlighted a potential negative relationship between cardiovascular pharmacological support and neonatal outcomes $(3,6,31,32)$. It remains unclear whether these findings relate to diagnostic imprecision or incorrect cardiovascular treatment choice, for example, vasopressor vs. inotrope. The results of our study reaffirmed early cardiovascular pharmacological support may be a marker for a clinical situation of increased risk of death and IVH, and these findings are consistent with results from the CNN (19) and the US study (9). Vasopressors may induce rapid cerebral hemodynamic changes, thereby impacting the germinal matrix, which may lead to $\operatorname{IVH}(33,34)$. Other studies have proposed that vasopressors may affect the selfregulation of cerebral vessels, leading to cerebral ischemia and reperfusion injury prior to IVH occurrence $(8,35)$. Besides, nonjudicious use of cardiovascular pharmacological support may also paradoxically augment the magnitude of left-right shunt of PDA by increasing systemic vascular resistance (36). In addition, several studies have demonstrated that although cardiovascular pharmacological support improve the numeric value of blood 
TABLE 4 | Outcomes and cardiovascular pharmacological support duration in different birth weight groups among survivors.

\begin{tabular}{|c|c|c|c|c|c|c|c|c|c|c|c|c|}
\hline \multirow[b]{2}{*}{ Outcomes } & \multicolumn{4}{|c|}{ Infants with birth weight $\geq 1,500 \mathrm{~g}$} & \multicolumn{4}{|c|}{ Infants with birth weight $1,000-1,500 \mathrm{~g}$} & \multicolumn{4}{|c|}{ Infants with birth weight $<1,000 \mathrm{~g}$} \\
\hline & $\begin{array}{c}\text { Duration } \leq 3 \\
\text { days } \\
N=393\end{array}$ & $\begin{array}{c}\text { Duration }>3 \\
\text { days } \\
N=660\end{array}$ & $\begin{array}{l}\text { Odds ratio } \\
\text { (95\% Cl) }\end{array}$ & $p$-value & $\begin{array}{c}\begin{array}{c}\text { Duration } \leq 3 \\
\text { days } \\
N=285\end{array} \\
N\end{array}$ & $\begin{array}{c}\text { Duration }>3 \\
\text { days } \\
N=637\end{array}$ & $\begin{array}{l}\text { Odds ratio } \\
\text { (95\% Cl) }\end{array}$ & $p$-value & $\begin{array}{c}\text { Duration } \leq 3 \\
\text { days } \\
N=44\end{array}$ & $\begin{array}{c}\text { Duration }>3 \\
\text { days } \\
N=136\end{array}$ & $\begin{array}{l}\text { Odds ratio } \\
\text { (95\% Cl) }\end{array}$ & $p$-value \\
\hline $\begin{array}{l}\text { Survival without } \\
\text { morbidity, } n / N(\%)\end{array}$ & $\begin{array}{c}332 / 393 \\
(84.5)\end{array}$ & $\begin{array}{c}528 / 660 \\
(80.0)\end{array}$ & $\begin{array}{c}1.36 \\
(0.96-1.93)\end{array}$ & 0.07 & $\begin{array}{c}191 / 285 \\
(67.0)\end{array}$ & $\begin{array}{c}381 / 637 \\
(59.8)\end{array}$ & $\begin{array}{c}1.37 \\
(1.01-1.85)\end{array}$ & 0.04 & 21/44 (48) & 47/136 (35) & $\begin{array}{c}1.73 \\
(0.82-3.64)\end{array}$ & 0.12 \\
\hline NEC, $n / N(\%)$ & $7 / 393(1.8)$ & 10/660 (1.5) & $\begin{array}{c}1.18 \\
(0.38-3.46)\end{array}$ & 0.74 & $15 / 285(5.3)$ & $32 / 637(5.0)$ & $\begin{array}{c}1.05 \\
(0.52-2.04)\end{array}$ & 0.88 & $2 / 44$ (5) & 12/136 (9) & $\begin{array}{c}0.49 \\
(0.05-2.36)\end{array}$ & 0.36 \\
\hline $\begin{array}{l}\text { Severe IVH }{ }^{\mathrm{a}}, n / \mathrm{N} \\
(\%)\end{array}$ & $8 / 358(2.2)$ & $36 / 631(5.7)$ & $\begin{array}{c}0.38 \\
(0.15-0.84)\end{array}$ & 0.01 & $20 / 275(7.3)$ & 72/630 (11.4) & $\begin{array}{c}0.61 \\
(0.34-1.04)\end{array}$ & 0.06 & $4 / 40(10)$ & 28/133 (21) & $\begin{array}{c}0.42 \\
(0.10-1.32)\end{array}$ & 0.11 \\
\hline$P V L^{b}, n / N(\%)$ & 8/349 (2.3) & 24/618 (3.9) & $\begin{array}{c}0.58 \\
(0.22-1.35)\end{array}$ & 0.18 & 8/267 (3.0) & $18 / 621(2.9)$ & $\begin{array}{c}1.03 \\
(0.38-2.54)\end{array}$ & 0.94 & $2 / 38(5)$ & 15/131 (11) & $\begin{array}{c}0.43 \\
(0.05-2.00)\end{array}$ & 0.26 \\
\hline ROP, n/N (\%) & 18/393 (4.6) & 29/660 (4.4) & $\begin{array}{c}1.04 \\
(0.54-1.98)\end{array}$ & 0.89 & 48/285 (16.8) & $\begin{array}{c}121 / 637 \\
(19.0)\end{array}$ & $\begin{array}{c}0.86 \\
(0.58-1.26)\end{array}$ & 0.44 & 16/44 (36) & 54/136 (40) & $\begin{array}{c}0.87 \\
(0.40-1.85)\end{array}$ & 0.69 \\
\hline $\begin{array}{l}\text { ROP } \geq \text { stage } 3 \\
n / N(\%)\end{array}$ & 0/393 (0) & 2/660 (0.30) & $0(0-3.23)$ & 0.28 & 4/285 (1.4) & 12/637 (1.9) & $\begin{array}{c}0.74 \\
(0.17-2.47)\end{array}$ & 0.61 & 6/44 (14) & 14/136 (10) & $\begin{array}{c}1.38 \\
(0.40-4.14)\end{array}$ & 0.54 \\
\hline BPD, $n / N(\%)$ & 32/393 (8.1) & 69/660 (10.5) & $\begin{array}{c}0.76 \\
(0.47-1.20)\end{array}$ & 0.22 & 56/285 (19.7) & $\begin{array}{c}140 / 637 \\
(22.0)\end{array}$ & $\begin{array}{c}0.87 \\
(0.60-1.24)\end{array}$ & 0.42 & $14 / 44$ (32) & 59/136 (43) & $\begin{array}{c}0.61 \\
(0.27-1.31)\end{array}$ & 0.17 \\
\hline LOS, $n / N(\%)$ & 10/393 (2.5) & 16/660 (2.3) & $\begin{array}{c}1.12 \\
(0.45-2.70)\end{array}$ & 0.78 & 19/285 (6.7) & 49/637 (7.7) & $\begin{array}{c}0.86 \\
(0.47-1.52)\end{array}$ & 0.58 & $1 / 44(2)$ & 25/136 (18) & $\begin{array}{c}0.10 \\
(0.002-0.68)\end{array}$ & 0.01 \\
\hline
\end{tabular}

NEC, necrotizing enterocolitis; IVH, intraventricular hemorrhage; PVL, periventricular leukomalacia; ROP, retinopathy of pre-maturity; BPD, bronchopulmonary dysplasia; LOS, culture-proven late onset sepsis.

alncidence of PVL or IVH was calculated among infants with neuroimaging results. Data on severe IVH were missing in 64, 18, 7 infants in 3 groups (birth weight $\geq 1,500 \mathrm{~g}$. birth weight 1,000-1,500 g, birth weight <1,000 g), respectively.

${ }^{b}$ Incidence of PVL or IVH was calculated among infants with neuroimaging results. Data on PVL were missing in 87, 36, 11 infants in 3 groups (birth weight $\geq 1,500 \mathrm{~g}$, birth weight 1,000-1,500 g, birth weight <1,000 g), respectively. 


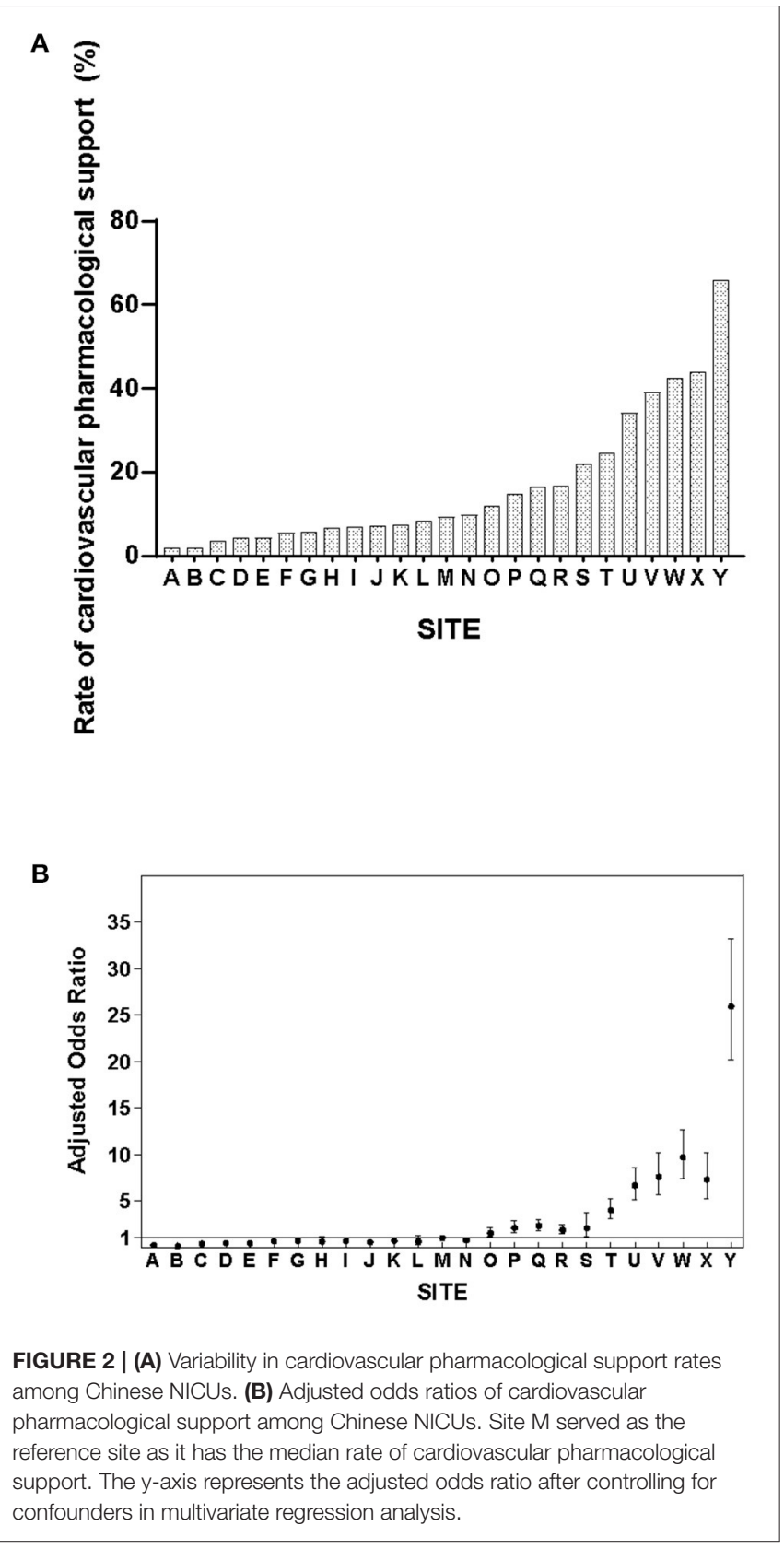

pressure, they may compromise systemic perfusion of vital organs and result in organ damage $(9,34,37)$. These data should not dissuade neonatologists against monitoring blood pressure; rather, it is incumbent of clinicians and hemodynamic scientists to identify critical blood pressure thresholds unique to relevant pathophysiologic states and evidence for disease-specific treatment strategies. We also found an increased risk of LOS in the cardiovascular pharmacological support group which may relate to sicker infants are more likely to have end-organ complications or receive steroids which may increase the risk of LOS.
Another striking finding was the high (63.1\%) rate of prolonged ( $>3$ days) cardiovascular pharmacological support compared to a median dopamine infusion time of $46 \mathrm{~h}$ per patient in NNN. We identified lower rates of "survival without morbidity" in VLBW infants who received prolonged cardiovascular pharmacological support. Although a major difference was also seen in ELBW and infants with birth weight $\geq 1,500$ infants, this was not statistically significant, which was most likely related to inadequate number of infants. The association between a high rate of cardiovascular pharmacological support in the early postnatal period and hypotension is thought to be related to impaired cardiac loading conditions due to the physiologic changes that occur in the transition environment $(2,38,39)$. Dempsey et al. found a spontaneous increase in blood pressure within $24 \mathrm{~h}$ after birth in ELBW infants who remained untreated (40). These findings were replicated by Batton et al. (41). These findings suggest that a shorter duration of cardiovascular pharmacological support might be sufficient for transient postnatal hypotension, if needed. There is still uncertainty regarding the optimal duration of cardiovascular pharmacological support (42).

Our study did not collect specific information on the type or dose of cardiovascular pharmacological support used for individual infants; however, a post-hoc survey concluded that dopamine was uniformly used as the first line agent. Definitely, there is considerable controversy in the choice of cardiovascular pharmacological support and the optimal drug in different situations. Besides, there is still uncertainty according to safety and effectiveness of vasopressor activity and inotropic activity drugs as for the insufficiency of pharmacology and pharmacodynamics information in these vulnerable group. It is noteworthy that dopamine has become the universal first line agent for hemodynamic instability, irrespective of disease state or ambient cardiovascular physiology, despite few data to support this trend. Dopamine appears to elevate blood pressure predominantly by vasoconstriction at the expense of systemic blood flow. It may have negative effects on hemodynamic stability, oxygen transport, cerebrovascular autoregulation, and cardiac output through an inotrope/vasopressor imbalance (43). For cardiovascular support in preterm infants, growing data suggest that dobutamine may be more appropriate during the transitional period than dopamine (33). Whether the adverse effects on neonatal outcomes in Chinese NICUs relate to the imprecision in the prolonged use of cardiovascular pharmacological support or non-judicious use of dopamine as the first line agent remains unknown. Previous study in US showed a declining trend in the use of dopamine and dobutamine and an increasing trend of the uses of hydrocortisone and vasopressin (1). These data highlight the importance to developed standardized clinical practice guidelines. The use of TnECHO might enhance diagnostic/therapeutic precision and provide additional details for longitudinal monitoring. Further quality improvement for cardiovascular pharmacological support could be made by standardizing decision-making process, redefining intervention framework of hypotension triggering therapies, improving antenatal corticosteroids provision, shortening the duration of cardiovascular pharmacological 
support and develop optimal management toward transient physiological blood pressure fluctuations.

Our study has several limitations. First, this study was based on a secondary analysis of a large prospective database, and the database did not include details about the type and dosage of cardiovascular pharmacological support. In addition, we did not collect data on delayed cord clamping, PDA, or receipt of surfactant, which may play a contributory role. Second, information about indications for medication initiation and termination was also not collected. Besides, data on the occurrence time of IVH was not available leading to difficulty in clarifying the association between cardiovascular pharmacological support and IVH. Third, our study only enrolled tertiary centers located in large metropolis cities, and the results might not represent the general status of cardiovascular pharmacological support in all levels of NICUs in China. Fourth, we only collected NICU-level data in our questionnaire, but unfortunately there might be variations of practices among different providers in the same NICU.

In conclusion, our results showed that cardiovascular pharmacological support are commonly used in Chinese NICUs, especially in extremely pre-mature infants and ELBW infants. Cardiovascular pharmacological support was associated with increased risks of death, severe IVH and BPD, and prolonged use may be associated with even worse outcomes. The rate of cardiovascular pharmacological support varied significantly between participating units. Future quality improvement efforts are urgently needed to facilitate the rational cardiovascular pharmacological support in Chinese NICUs.

\section{DATA AVAILABILITY STATEMENT}

The raw data supporting the conclusions of this article will be made available by the authors, without undue reservation.

\section{REFERENCES}

1. Rios DR, Moffett BS, Kaiser JR. Trends in pharmacotherapy for neonatal hypotension. J Pediatr. (2014) 165:697701.e1. doi: 10.1016/j.jpeds.2014.06.009

2. Wu TW, Azhibekov T, Seri I. Transitional hemodynamics in preterm neonates: clinical relevance. Pediatr Neonatol. (2016) 57:7-18. doi: 10.1016/j.pedneo.2015.07.002

3. Faust K, Hartel C, Preuss M, Rabe H, Roll C, Emeis M, et al. Shortterm outcome of very-low-birthweight infants with arterial hypotension in the first 24 h of life. Arch Dis Child Fetal Neonatal Ed. (2015) 100:F38892. doi: 10.1136/archdischild-2014-306483

4. Batton BJ, Li L, Newman NS, Das A, Watterberg KL, Yoder BA, et al. Feasibility study of early blood pressure management in extremely preterm infants. J Pediatr. (2012) 161:65-9.e1. doi: 10.1016/j.jpeds.2012.01.014

5. Dempsey EM, Barrington KJ. Diagnostic criteria and therapeutic interventions for the hypotensive very low birth weight infant. $J$ Perinatol. (2006) 26:677-81. doi: 10.1038/sj.jp.7211579

6. Pellicer A, Bravo MC, Madero R, Salas S, Quero J, Cabanas F. Early systemic hypotension and vasopressor support in low birth weight infants: impact on neurodevelopment. Paediatrics. (2009) 123:1369-76. doi: 10.1542/peds.2008-0673

7. Durrmeyer X, Marchand-Martin L, Porcher R, Gascoin G, Roze JC, Storme L, et al. Abstention or intervention for isolated hypotension in the first 3 days

\section{AUTHOR CONTRIBUTIONS}

NL conceptualized, designed the study, analyzed, interpreted data, drafted the manuscript, and performed statistical analyses. SJ conceptualized, designed the study, acquired, analyzed, interpreted data, revised the manuscript, and performed statistical analyses. PM analyzed, interpreted data, reviewed, and revised the manuscript for important intellectual content. $\mathrm{XL}, \mathrm{YG}, \mathrm{YW}$, and YY interpreted data, reviewed, and revised the manuscript for important intellectual content. JH and YD acquired, interpreted data, reviewed, and revised the manuscript for important intellectual content. SL and YC conceptualized, designed the study, analyzed, interpreted data, reviewed, and revised the manuscript for important intellectual content and supervised the study. All authors approved the final manuscript as submitted and agree to be accountable for all aspects of the work.

\section{FUNDING}

This study was funded by the China Medical Board (Grant Number 14-194) and the Canadian Institutes of Health Research (Grant Number CTP 87518).

\section{ACKNOWLEDGMENTS}

We would like to express our gratitude to all the units that took part in this study and their principals.

\section{SUPPLEMENTARY MATERIAL}

The Supplementary Material for this article can be found online at: https://www.frontiersin.org/articles/10.3389/fped. 2021.638540/full\#supplementary-material of life in extremely preterm infants: association with short-term outcomes in the EPIPAGE 2 cohort study. Arch Dis Child Fetal Neonatal Ed. (2017) 102:490-6. doi: 10.1136/archdischild-2016-312104

8. Abdul AA, Thomas S, Murthy P, Rabi Y, Soraisham A, Stritzke A, et al. Early inotropes use is associated with higher risk of death and/or severe brain injury in extremely premature infants. J Matern Fetal Neonatal Med. (2019) 33:2751-758. doi: 10.1080/14767058.2018.1560408

9. Batton B, Li L, Newman NS, Das A, Watterberg KL, Yoder BA, et al. Early blood pressure, antihypotensive therapy and outcomes at 18-22 months' corrected age in extremely preterm infants. Arch Dis Child Fetal Neonatal Ed. (2016) 101:F201-6. doi: 10.1136/archdischild-2015-308899

10. Group RS. Reduction of infection in neonatal intensive care units using the evidence-based practice for improving quality (REINEPIQ): a study protocol. Chin J Evid Based Pediatr. (2018) 13:64-9. doi: 10.3969/j.issn.1673-5501.2018.01.010

11. Jiang S, Yan W, Li S, Zhang L, Zhang Y, Shah PS, et al. Mortality and morbidity in infants $<34$ weeks' gestation in 25 NICUs in China: a prospective cohort study. Front Pediatr. (2020) 8:33. doi: 10.3389/fped.2020.00033

12. Lee SK, Aziz K, Dunn M, Clarke M, Kovacs L, Ojah C, et al. Transport risk index of physiologic stability, version II (TRIPS-II): a simple and practical neonatal illness severity score. Am J Perinatol. (2013) 30:395400. doi: 10.1055/s-0032-1326983

13. Papile LA, Burstein J, Burstein R, Koffler H. Incidence and evolution of subependymal and intraventricular hemorrhage: a study of infants 
with birth weights less than 1,500 gm. J Pediatr. (1978) 92:52934. doi: 10.1016/S0022-3476(78)80282-0

14. International Committee for the Classification of Retinopathy of Prematurity. The international classification of retinopathy of prematurity revisited. Arch Ophthalmol. (2005) 123:991-9. doi: 10.1001/archopht.123.7.991

15. Jobe AH, Bancalari E. Bronchopulmonary dysplasia. Am J Respir Crit Care Med. (2001) 163:1723-9. doi: 10.1164/ajrccm.163.7.2011060

16. Gastmeier P, Geffers C, Schwab F, Fitzner J, Obladen M, Rüden H. Development of a surveillance system for nosocomial infections: the component for neonatal intensive care units in Germany. J Hosp Infect. (2004) 57:126-31. doi: 10.1016/j.jhin.2003.12.038

17. Jajoo M, Manchanda V, Chaurasia S, Sankar MJ, Gautam H, Agarwal R. Investigators of the Delhi neonatal infection study (DeNIS) collaboration, New Delhi, India. Alarming rates of antimicrobial resistance and fungal sepsis in outborn neonates in North India. PLoS ONE. (2018) 13:e0180705. doi: 10.1371/journal.pone.0180705

18. Walsh MC, Kliegman RM. Necrotizing enterocolitis: treatment based on staging criteria. Pediatr Clin N Am. (1986) 33:179201. doi: 10.1016/S0031-3955(16)34975-6

19. Wong J, Shah PS, Yoon EW, Yee W, Lee S, Dow K. Inotrope use among extremely preterm infants in Canadian neonatal intensive care units: variation and outcomes. Am J Perinatol. (2015) 32:9-14. doi: 10.1055/s-0034-1371703

20. Lasky T, Greenspan J, Ernst FR, Gonzalez L. Dopamine and dobutamine use in preterm or low birth weight neonates in the premier 2008 database. Clin Ther. (2011) 33:2082-8. doi: 10.1016/j.clinthera.2011.11.001

21. Sehgal A, Osborn D, McNamara PJ. Cardiovascular support in preterm infants: a survey of practices in Australia and New Zealand. J Paediatr Child Health. (2012) 48:317-23. doi: 10.1111/j.1440-1754.2011.02246.x

22. Al-Aweel I, Pursley DM, Rubin LP, Shah B, Weisberger S, Richardson DK. Variations in prevalence of hypotension, hypertension, and vasopressor use in NICUs. J Perinatol. (2001) 21:272-8. doi: 10.1038/sj.jp.7210563

23. Burns ML, Stensvold HJ, Risnes K, Guthe HJ, Astrup H, Nordhov SM, et al. Inotropic therapy in newborns, a populationbased national registry study. Pediatr Crit Care Med. (2016) 17:948-56. doi: 10.1097/PCC.0000000000000898

24. Laughon M, Bose C, Allred E, O'Shea TM, Van Marter LJ, Bednarek F, et al. Factors associated with treatment for hypotension in extremely low gestational age newborns during the first postnatal week. Pediatrics. (2007) 119:273-80. doi: 10.1542/peds.2006-1138

25. Garvey AA, Kooi E, Dempsey EM. Inotropes for preterm infants: 50 years on are we any wiser? Front Pediatr. (2018) 6:88. doi: 10.3389/fped.2018.00088

26. Kent AL, Meskell S, Falk MC, Shadbolt B. Normative blood pressure data in non-ventilated premature neonates from 28-36 weeks gestation. Pediatr Nephrol. (2009) 24:141-6. doi: 10.1007/s00467-008-0916-9

27. Travers CP, Clark RH, Spitzer AR, Das A, Garite TJ, Carlo WA. Exposure to any antenatal corticosteroids and outcomes in preterm infants by gestational age: prospective cohort study. BMJ. (2017) 356:j1039. doi: 10.1136/bmj.j1039

28. Liu X. The morbidities of extremely preterm and extremely low birth weight infants during hospitalization. Zhonghua Er Ke Za Zhi. (2015) 53:334-40. doi: 10.3760/cma.j.issn.0578-1310.2015.05.005

29. Kong $\mathrm{X}, \mathrm{Xu} F, \mathrm{Wu} \mathrm{R}, \mathrm{Wu} \mathrm{H}$, Ju R, Zhao X, et al. Neonatal mortality and morbidity among infants between 24 to 31 complete weeks: a multicenter survey in China from 2013 to 2014. BMC Pediatr. (2016) 16:174. doi: 10.1186/s12887-016-0716-5

30. Zhu J, Xie Y, Wang B, Wang Y, Akinbi H, Xie L. Epidemiological investigation on the current practice of umbilical cord clamping in China. Am J Perinatol. (2020). doi: 10.1055/s-0040-1721494. [Epub ahead of print].
31. Kuint J, Barak M, Morag I, Maayan-Metzger A. Early treated hypotension and outcome in very low birth weight infants. Neonatology. (2009) 95:3116. doi: 10.1159/000180113

32. Kurtom W, Jain D, Quan M, Vanbuskirk S, Bancalari E, Claure N. The impact of late onset arterial hypotension on respiratory outcome in extremely premature infants. Neonatology. (2019) 115:164-8. doi: 10.1159/000494104

33. Seri I. Cardiovascular support in the preterm: treatments in search of indications. J Pediatr. (2007) 150:e31-3; author reply e33. doi: 10.1016/j.jpeds.2006.09.023

34. Noori S, Stavroudis TA, Seri I. Systemic and cerebral hemodynamics during the transitional period after premature birth. Clin Perinatol. (2009) 36:72336.v. doi: 10.1016/j.clp.2009.07.015

35. Noori S, Seri I. Hemodynamic antecedents of peri/intraventricular hemorrhage in very preterm neonates. Semin Fetal Neonatal Med. (2015) 20:232-7. doi: 10.1016/j.siny.2015.02.004

36. Zhang J, Penny DJ, Kim NS, Yu VY, Smolich JJ. Mechanisms of blood pressure increase induced by dopamine in hypotensive preterm neonates. Arch Dis Child Fetal Neonatal Ed. (1999) 81:F99-104. doi: 10.1136/fn.81.2.F99

37. Golder V, Hepponstall M, Yiallourou SR, Odoi A, Horne RS. Autonomic cardiovascular control in hypotensive critically ill preterm infants is impaired during the first days of life. Early Hum Dev. (2013) 89:41923. doi: 10.1016/j.earlhumdev.2012.12.010

38. Hooper SB, Te PA, Lang J, van Vonderen JJ, Roehr CC, Kluckow M, et al. Cardiovascular transition at birth: a physiological sequence. Pediatr Res. (2015) 77:608-14. doi: 10.1038/pr.2015.21

39. Dilli D, Soylu H, Tekin N. Neonatal hemodynamics and management of hypotension in newborns. Turk Pediatri Ars. (2018) 53:S65-75. doi: 10.5152/TurkPediatriArs.2018.01801

40. Dempsey EM, Al HF, Barrington KJ. Permissive hypotension in the extremely low birthweight infant with signs of good perfusion. Arch Dis Child Fetal Neonatal Ed. (2009) 94:F241-4. doi: 10.1136/adc.2007. 124263

41. Batton B, Li L, Newman NS, Das A, Watterberg KL, Yoder BA, et al. Evolving blood pressure dynamics for extremely preterm infants. J Perinatol. (2014) 34:301-5. doi: 10.1038/jp.2014.6

42. Dempsey EM. What should we do about low blood pressure in preterm infants. Neonatology. (2017) 111:402-7. doi: 10.1159/000460603

43. Dempsey EM, Kooi EM. Blood pressure management in the very preterm infant: more than just millimetres. In: Cusack J, Boyle EM, editors. Emerging Topics and Controversies in Neonatology. Berlin: Springer (2020). p.289-306.

Conflict of Interest: SL received support for article research from the Canadian Institutes of Health Research (CIHR). YC institution received funding from China Medical Board and CIHR.

The remaining authors declare that the research was conducted in the absence of any commercial or financial relationships that could be construed as a potential conflict of interest.

Copyright $\odot 2021$ Luo, Jiang, McNamara, Li, Guo, Wang, Han, Deng, Yang, Lee and Cao. This is an open-access article distributed under the terms of the Creative Commons Attribution License (CC BY). The use, distribution or reproduction in other forums is permitted, provided the original author(s) and the copyright owner(s) are credited and that the original publication in this journal is cited, in accordance with accepted academic practice. No use, distribution or reproduction is permitted which does not comply with these terms. 